\title{
Mutineries Armées et Réversibilité du Sentiment de Sécurité chez les Populations Civiles: Une Analyse à Partir des « grins » de Bouaké en Côte d'Ivoire
}

\author{
Silué N'Tchabétien Oumar, \\ Université Alassane Ouattara de Bouaké, Côte d'Ivoire
}

Doi:10.19044/esj.2019.v15n29p138 URL:http://dx.doi.org/10.19044/esj.2019.v15n29p138

\section{Résumé}

Cet article questionne le lien entre les pratiques des armées africaines et la réversibilité du sentiment de sécurité chez les civils. Plus précisément, à partir d'une enquête en cours depuis janvier 2017, l'article montre comment l'armée ivoirienne a créé chez les civils une grande mobilité du sentiment de sécurité qui oscille entre l'absence et/ou la réduction de menaces et un immense sentiment de vulnérabilité en présence du 'corps habillé".

Mots-clés: Armées, Mutinerie, Côte d'Ivoire, Grins, Violence

\section{Armed Mutinies and Reversibility of the Sense of Security among Civilian Populations: An Analysis from the "Grins" of Bouaké in Côte d'Ivoire}

\section{Silué N'Tchabétien Oumar,}

Université Alassane Ouattara de Bouaké, Côte d'Ivoire

\begin{abstract}
This paper focuses on questioning the link between the practices of armed forces and the reversibility of the sense of security among civilians. Specifically, based on a survey on its progress since January 2017, this paper shows how the Ivorian army has created a high mobility of the sense of security among civilians. This oscillates between the absence and/or reduction of threats and an immense sense of vulnerability in the presence of the "dressed bodies".
\end{abstract}


Keywords: Armies, Mutiny, Côte d'Ivoire, Grins, Violence

\section{Introduction}

A sa Une du 18 au 24 mai $2017^{5}$, le journal satirique Gbich titrait «Accord bancal est mieux que froufrou » avec en photo, le Président Alassane Ouattara, l'air hagard et débout, mis en joue par un soldat mutin tenant un fusil kalachnikov modifié avec une main portant un gant blanc gant blanc. Avec un ton humoristique, ce journal peint le tableau de l'actualité de l'heure en Côte d'Ivoire: la mutinerie des soldats. Le soulèvement des militaires vient s'ajouter à la longue liste des événements violents qui traversent la Côte d'Ivoire depuis la disparition d'Houphouët-Boigny en 1993.

Le microcosme socio-politique ivoirien subi depuis le milieu des années 90 des chocs violents. Le 24 décembre 1999, des soldats se soulèvent et déposent le Président Henri Konan Bédié. Dirigée par le Général Guéï Robert la junte militaire prétend être venue pour «balayer la maison » et restituer le pouvoir aux civils avant la fin de l'année 2000 (Akindès, 2008, p.39). Le coup de force des « jeunes gens » dirigés par Guéï Robert vise selon les responsables du Conseil National de Salut Public (CNSP) ${ }^{6}$, à assainir les dépenses publiques, la restauration des droits politiques d'Alassane Ouattara et des autres exilés politiques, etc.

En 2000, Laurent Gbagbo accède au pouvoir dans des circonstances complexes. En effet, dans son refus de céder le pouvoir, la junte militaire organise une action de répression des civils notamment, des militants et sympathisants du Front Populaire Ivoirien (FPI) déterminés à ne pas se faire ravir leur victoire. Les violences ont provoqué plusieurs victimes.

L'accession de Gbagbo Laurent au pouvoir n'a pas mis fin aux violences. Pour couper la chaîne, le pouvoir a organisé en 2001 une immense opération de réconciliation nationale. L'objet était de réunir tous ceux qui se sont opposés d'une manière ou d'une autre pour construire une catharsis de pardon. Ouvert le 9 octobre 2001, le Forum de réconciliation nationale a duré 2 mois. Le Forum s'est achevé par 14 résolutions qui visaient à ramener la paix. Il s'agissait pour les participants de résoudre les problèmes de la nationalité d'Alassane Ouattara, la réouverture d'une enquête sur les circonstances entourant la mort de 57 hommes dont les corps ont été découverts à Yopougon, une enquête sur les circonstances du coup d'Etat du 24 décembre 1999 qui a occasionné la perte du pouvoir par Henri Konan Bédié, la réforme du système judiciaire et de la sécurité, etc. Mais les grosses dépenses et la débauche d'énergie mobilisées ne viendront pas à bout des ressentiments et des rancunes. Le 19 septembre 2002, un énième conflit

${ }^{6} \mathrm{C}$ 'est un organe mis en place par les militaires pour assurer la gestion des affaires de l'Etat. 
militaro-politique éclate. La Côte d'Ivoire sera divisée pendant 10, partagé entre le nord contrôlé par la rébellion, les Forces Armées des Forces Nouvelles (FAFN), et le sud sous la surveillance des forces loyalistes, les Forces De Défense et de Sécurité (FDS).

En 2010, des élections sont organisées pour ramener définitivement le calme en conférant au futur chef d'Etat une solide légitimité. Mais elles débouchent en une violente crise post-électorale qui a fait plusieurs victimes. Laurent Gabgbo, élu selon le Conseil constitutionnel avec 51,45\% contre 48,55\% pour Alassane Ouattara. Au contraire, la Commission Electorale Indépendante (CEI) déclare Alassane Ouattara avec 54,10\% contre 45,90\% pour Alassane Ouattara. L'arrivée au pouvoir d'Alassane Ouattara en 2011 a été suivi par un vaste programme de réformes dans plusieurs secteurs dont celui de la sécurité à travers la Réforme du Secteur de la Sécurité (RSS). L'objet est de mettre en œuvre un train de mesures pour intégrer les combattants qui ont pris les armes entre 2002 et 2010. Les mécanismes d'intégration relèvent des partenaires au développement ou de l'Etat ivoirien. En dépit des campagnes médiatiques autour des programmes d'insertion des ex-combattants, la Côte d'Ivoire a été confronté à des mutineries entre 2014 et 2017. Qui sont les soldats qui se mutinent? Quelles sont les motivations des soldats mécontents et quelles sont les logiques à l'œuvre ? Les populations civiles sont-elles rassurées par les soldats ? Comment-vivent-elles ces coups de force?

Cet article se propose de questionner la réversibilité du sentiment de sécurité chez les populations civiles en butent aux soulèvements des soldats. Les données ont été collectées grâce à des observations et des entretiens auprès de 32 personnes issus de deux «grins ${ }^{7}$ » à Bouaké : le «grin» «les intellectuels » au quartier N'gattakro et un autre à N'dakro. Le choix de la ville de Bouaké tient du fait que, ancienne base de la rébellion, elle a abrité le quartier général des FAFN entre 2002 et 2010. Depuis 2011, cette ville fonctionne également comme le siège de toutes les contestations violentes liées à l'armée ivoirienne et parfois même de la société civile.

L'article s'ouvre par une présentation des soldats mutinés en mettant un accent sur leur identité. Cette partie présente également le déroulement des différentes mutineries. Ensuite la seconde partie présente la réversibilité du sentiment de sécurité chez les membres des « grins ». Enfin, la dernière partie analyse les implications de la réversibilité du sentiment de sécurité.

\footnotetext{
${ }^{7}$ Le grin est un lieu où des individus se retrouvent pour discuter. Les débats sont accompagnés par la consommation de thé et parfois de victuailles comme des arachides, de la viande, etc.
} 


\section{Trajectoires croisées des soldats : les 8400 et les « démos »}

Les hommes armés qui se sont soulevés sont tous issus de la rébellion de 2002 en Côte d'Ivoire. Ils sont apparus lors de la mise en œuvre du programme de DDR dans le cadre de l'Accord Politique de Ouagadougou (APO). Le 4 mars 2007, le Président Laurent Gbagbo et Soro Kigbafori Guillaume, chef de la rébellion et le Président burkinabé, Blaise Compaoré se retrouvent pour tenter de régler la crise ivoirienne. Il porte sur le processus électoral, le désarmement et la réunification de la Côte d'Ivoire.

C'est sur la base de l'APO que les anciens combattants de la zone Centre, Nord et Ouest (CNO), contrôlée par la rébellion vont se loger dans le processus. Les anciens rebelles suivent un itinéraire bien cadré : regroupement, désarmement, visite médicale, profilage et démobilisation qui s'achèvent par l'identification et l'acquisition d'une carte de démobilisée des FAFN. Le processus avait pour but de séparer les combattants en deux catégories : celle des combattants toujours aptes à exercer le métier des armes selon le critère de l'âge, de la santé, etc. et celle de ceux qui sont susceptibles d'être renvoyés à la vie civile.

La première catégorie est celle des personnes (hommes et femmes) qui, remplissant les critères d'aptitude au métier des armes, sont intégrés à l'armée. Ils sont au nombre de 8400 . Ils se retrouvent principalement chez les militaires. Ils ont été reversés dans les différentes unités de l'armée ivoirienne.

La seconde catégorie est celles des démobilisés ou «démos » ou encore la "cellule 39'. Ce sont les anciens combattants qui sont retournés à la vie civile. Ils ont bénéficié de mesures d'insertion professionnelles. Ils ont bénéficié d'un accompagnement dans l'élevage, l'agriculture, le commerce, etc.

Les anciens éléments des FAFN qui se sont mutés sont les soldats reversés dans l'armée. Du 6 au 7 janvier 2017, ils ont occupé plusieurs villes (Bouaké, Abidjan, Man, Korhogo, Bouna, etc.) pour réclamer des primes ECOMOG et l'amélioration de leurs conditions de travail ${ }^{8}$. Après deux jours de grande frayeur les militaires perçoivent 5 millions de Fcfa sous réserve de percevoir le reliquat au mois de mai. Mais l'efficacité des soldats mutins provoque l'ire des démobilisés qui menacent de rentrer aussi en dissidence par la force ${ }^{9}$.

Du 12 au 15 mai 2017, les soldats remettent le couvert. Ils lancent un coup de force plus violent que le précédent. On dénombre plusieurs blessés et 3 morts officiellement. A l'issue d'un «accord » frappé du sceau du secret défense entre le gouvernement et les mutins, les soldats perçoivent encore 5 millions avec la garantie de recevoir le reliquat de 2 millions fin mai.

\footnotetext{
${ }^{8}$ Soir info, $\mathrm{N}^{\circ} 6676$ du 10 janvier 2017.

${ }^{9}$ L'Inter, $\mathrm{N}^{\circ} 5580$ du mercredi 25 janvier 2017.
} 


\section{La réversibilité du sentiment de sécurité}

Le mieux-être ou le mal être sont les deux sentiments que les soldats suscitent auprès des populations. Par le biais de la notion de réversibilité, on lit la mobilité du sentiment créé par le rapport du civil au soldat. Dans les deux «grins » les deux sentiments transparaissaient dans les propos des membres. Le "grin" se résume à quelques bancs et des chaises disposées en forme rectangulaire ou circulaire. Quelques tabourets viennent compléter ces sièges. Deux théières, un brasero, un éventail de fortune (en vieux morceaux de planche ou de papier ou même une assiette), des verres (un grand et trois ou quatre petits), du sucre en poudre et de l'eau constituent le matériel de préparation du thé.

Pour les membres des «grin », le soldat mutin ne rassure pas. Il est source d'insécurité. Un membre du «grin » les intellectuels de N'gattakro affirme que :

«Les mutins nous fatiguent. Quand ils sortent comme ça on a peur. Ils tirs n'importe comment et si balle-là te prend tu peux mourir ». [K., 8 janvier 2017]

Un autre membre dit la même chose en mettant en cause le rôle de protecteur du soldat dévoyé. Il dit à ce sujet :

"Ceux-là c'est pour tuer les gens ici. Quand ils sont en train de faire leurs conneries ils blessent et ils tuent. On peut même pas travailler. Nous les vendeurs quand ils tirs on est obligé de fermé et on rentre les poches vides ». [C., 8 janvier 2017]

$\mathrm{Si}$, la plupart des membres des « grins » perçoivent le soldat mutiné comme une menace, il n'en demeure pas moins qu'à bien des égards, d'autres sentiments d'adhésion apparaissent. En effet, même s'ils ne sont pas en nombre important, certains d'entre eux, perçoivent les mutins comme des héros. Les traits de héros transparaissent dans leurs pratiques discursives.

«Ah mon frère ceux-là se sont nos frères aussi. On a nos parents frères sœurs dans les mutins là aussi ». [S., 11 mai 2017]

$\mathrm{Au}$ de-là de la parenté, on note des sentiments de sécurité aux accents politiques chez les membres.

"Même si ils déconnent parfois les gars là on peut pas parler

trop. Ce sont eux qui nous ont sauvé. Si eux ils n'avaient pas pris

les armes là, nous on était foutu ». [Y., 11 mai 2017]

On le voit, la réversibilité du sentiment de sécurité chez les populations est liée non seulement à l'idéologie et à la relation mais aussi par la situation. Cette situation change selon la conjoncture. 


\section{Armée ivoirienne : militaire ou mercenaire}

La situation de l'armée ivoirienne ouvre la voie à plusieurs analyses. On peut avancer que la «greffe » que le gouvernement a tenté de faire en mettant ensemble des anciens FDS et les FAFN n'a pas pris. Les anciens rebelles sont toujours prisonniers de leur mépris pour les FDS. Ce mépris est d'autant plus important qu'il concerne, pour les FDS, des personnes peu instruites qui ont intégré l'armée à la faveur d'une crise militaire. En effet, la plupart d'entre eux expriment un fort attachement à leurs anciennes unités. Cet attachement est aussi lié aux anciens commandants de zones. Les anciens FDS sont comme des fantômes qui errent dans un site.

Aussi, les mutineries à répétitions laissent transparaître l'indiscipline qui gangrène les Forces Armées de Côte d'Ivoire (FACI). Les anciens rebelles n'ont pas les valeurs de discipline et d'honneur des militaires. En plus, les mutins révèlent le faible niveau de formation des soldats. Leur comportement frise celui de mercenaires prêts au coup de feu pour sa récompense.

Le lien entre l'armée et la population civile est lâche voire inexistant. Le soldat qui rassure et qui protège a laissé la place à un mercenaire, un soldat dévoyé sans foi ni loi. L'armée fonctionne comme une menace qui met en péril la sécurité des ivoiriens.

Au-delà du registre militaire, les violences exercées par les militaires sur les membres du Rassemblement Des Républicains (RDR) à Bouaké marque la rupture entre les anciens alliés d'hier. La séparation entre le parti au pouvoir et l'armée qui l'a porté à la magistrature suprême. C'est une sorte de réversibilité dans la mesure où on note une rupture, une césure voire un violent déchirement entre deux alliés.

La séparation de l'armée au RDR est un mauvais présage pour le régime de Ouattara. La plaie provoquée peut s'étendre aux militants déçus et désabusés. Ce qui compromet l'avenir politique du $\mathrm{RDR}^{10}$.

\section{Conclusion}

L'armée ivoirienne est traversée par des crises à répétition depuis les années 1990. Les initiatives pour restaurer l'ordre n'ont pas abouti.

Pis les soldats, à la faveur des crises se sont démobilisés et ont versés dans l'indiscipline. Cette indiscipline se traduit dans les mutineries qui minent la Côte d'Ivoire depuis peu.

Dans les «grins », la réversibilité du sentiment de sécurité est complexe. Il oscille entre le sentiment de sécurité et celui d'insécurité. Au-

\footnotetext{
${ }^{10}$ OFPRA, 2017, Les anciens com'zones dans l'armée ivoirienne. Trafics, corruption, zones d'influence. [En ligne] Disponible sur :

https://www.ofpra.gouv.fr/sites/default/files/atoms/files/19_civ_armee_de_comzones.pdf (consulté le 4 avril 2019]
} 
delà de la réversibilité, la mutinerie traduit le mal être de la Côte d'Ivoire postcrise.

\section{References:}

1. Akindès, F. (2000). 'Racines des crises socio-politiques en Côte d'Ivoire et sens de l'histoire', in Ouédraogo, J-B., et Sall, E., Dir, Frontières de la citoyenneté et violence politique en Côte d'Ivoire, pp. 25-62.

2. Banegas, R. \& Marshall-Fratani, N. (2003). 'La Côte d'Ivoire en guerre : dynamiques du dedans et du dehors" in Politique Africaine (89).

3. Dembélé, O. (2002). 'La construction économique et politique de la catégorie "'étranger" en Côte d'Ivoire" in Côte d'Ivoire. L'année terrible 1999-2000. M Le Pape and Vidal C, pp ; 123-172.

4. Dembélé, O. (2003). "Côte d'Ivoire : La fracture communautaire" in Politique Africaine (78) : 34-48.

5. Kieffer, G. A. (2000). "Armée ivoirienne : le refus du déclassement"' in Politique Africaine, №78, pp.26-44.

6. Kieffer, J. (2006). 'Les jeunes des "grins' de thé et la campagne électorale de Ouagadougou' in Politique Africaine, $\mathrm{N}^{\mathrm{o}} 101, \mathrm{pp} .63-82$.

7. Soro, G. (2005). Pourquoi je suis devenu un rebelle, La Côte d'Ivoire au bord du gouffre, Paris, Hachette Littératures, 173 p. 